\title{
Terahertz Imaging Technique for Cancer Diagnostics Using Frequency Conversion by Gold Nano-Objects
}

\author{
K.A. Moldosanov ${ }^{1}, \underline{\text { A.V. Postnikov }}{ }^{2}$, V.M. Lelevkin ${ }^{1}$ and N.J. Kairyev ${ }^{1}$ \\ ${ }^{1}$ Kyrgyz-Russian Slavic University, 44 Kiyevskaya St., Bishkek 720000, Kyrgyzstan \\ E-mail : altair1964@yandex.ru \\ ${ }^{2}$ Université de Lorraine, LCP-A2MC, 1 Bd Arago, F-57078 Metz, France \\ E-mail : andrei.postnikov@univ-lorraine.fr
}

A technique is suggested (that includes the device's scheme and assessments of its feasibility) for imaging cancer cells in biological tissue samples in vitro and in human skin in vivo, exposing them to $4.2 \mathrm{THz}$ radiation and using a THz-to-infrared (IR) converter in combination with an IR sensitive camera. The converter includes a system of gold nanoparticles embedded into a substance transparent in THz and IR, e.g., Teflon ${ }^{\circledR}$. The heat exchange between gold nanoparticles and their embedding substance is simulated in a calculation. The convenient size of nanoparticles and their concentration in the substrate are estimated. 


\section{Introduction}

The terahertz $(\mathrm{THz})$ band of the electromagnetic spectrum extends from upper edge of microwaves to near infrared. $\mathrm{THz}$ radiation is non-ionizing, it can penetrate beneath the human skin, possessing the potential to spot tumours, and is considered safe for the public to be surveyed.

The tumour's visualising is based on the THz radiation's sensitivity to water, one of the most important components of the biological tissue. Ни и Nuss [1] were likely the first to demonstrate the biomedical $\mathrm{THz}$ imaging; they showed that different water content in different tissues (e.g., porcine muscle and fat) could provide the necessary contrast. Even earlier, a difference was indicated $[2,3]$ in the water content between normal tissues and cancerous tumours, namely, that the cancer cells contained more water. The fact that the water molecules absorb in the entire $\mathrm{THz}$ band (0.1-10 THz) [4] motivated a THz imaging of cancer cells in transition geometry (for in vitro study of thin, clinically prepared tissue sample). An elevated water content in cancer cells in combination with the fact that the $\mathrm{THz}$ waves cannot penetrate moist tissue favoured the development of $\mathrm{THz}$ medical imaging in reflection geometry (for investigations in vivo), whereas the transition geometry seems applicable for studying the samples of any body organ.

The contrast of recognising the cancer cells can be further enhanced by heating the water contained therein [5]. To this end, gold nanoparticles (GNPs) conjugated with biomolecules like antibodies are placed either directly into the patient's tumour, or into the bloodstream. In the latter case, on having got into the blood vessels feeding the tumour, the GNPs, by mediation of the antibodies, stick to the surface of the cancer cells, ignoring the healthy ones. The heating of GNPs and hence the water in the cells can be brought about by exposing the 
tissue to near-infrared (NIR) laser beam (at wavelengths $\sim 650-1350 \mathrm{~nm}$, that is the so-called "therapeutic window" characterised by maximum penetration depth of light in tissue). In GNPs under irradiation, the surface plasmons are excited, which, in the process of being damped out, heat the surrounding water. Consequently the cancer cells reflect the incident $\mathrm{THz}$ radiation stronger. Obviously, this method of enhancing the contrast by heating via delivered GNPs is only applicable in vivo and hence in the reflection geometry.

The issues of plasmon generation, thence resulting heating and the enhanced contrast in $\mathrm{THz}$ are covered by a series of contributions by Oh et al. [6-10]. The heating of GNPs and, consequently, the cancer cells can be used not only for increasing the contrast of tissues in $\mathrm{THz}$, but also for treatment of cancer by hyperthermia. In the context of related applications for diagnostics and therapy, one speaks of theranostics. The realisation of any imaging setup demands two essential elements: the radiation source and the detector. Among those available in the THz range, there are not many that would combine robustness, sufficient performance / sensitivity and affordable price for large-scale application. At the same time, a considerable apparatus' size is not necessarily an obstacle. In a recent work [11], two of us suggested a principle of $\mathrm{THz}$ generation by an array of gold nano-bars or nano-rings exposed to irradiation in the microwave range. The principle of operation involved is very briefly explained in Section 2. The THz to infrared (IR) conversion part, outlined in Section 3, is independent on how the primary $\mathrm{THz}$ radiation was actually generated; however, it makes reference to physical principles related to those addressed in Section 2. Both mechanisms deal with diversion of energy between different channels via an interplay with electron excitations and absorption of radiation quanta. Numerical estimates concerning the efficiency of THz to IR transformation by embedded nanoparticles are concluded by a short discussion. 


\section{Terahertz radiation source}

The idea outlined in [11] is to make use of the energy "bath" of longitudinal acoustic (LA) phonons which "live" in bulk crystals and in nanoparticles; the peak of their energy in gold spans the range of energies $\approx 16-19 \mathrm{meV}$, i.e. frequencies $3.9-4.6 \mathrm{THz}$. The trick to "liberate" the energy of lattice vibrations and divert it into that of released $\mathrm{THz}$ quanta consists of nearly matching the related energy step with the interval between the discrete electron levels, quantified due to a spatial confinement. Hence the need for nano-objects as essential elements of the setup. In an array of correspondingly "tuned" GNPs exposed to microwave radiation (hence at much lower frequencies than those of THz-range phonon or electron transitions), the phonon-assisted electron excitations may take place, whereof the subsequent relaxation would be primarily "diverted" into emitting a THz quantum rather than releasing the energy back to the phonon bath. The key element in controlling the preferential "diversion scheme" is the nano-objects's shape; it is argued in Ref. [11], after considering possible scenarios for the electron relaxation, that choosing either elongated (nano-bars) or circular (nano-rings) objects would do the trick. The estimates of the favourable objects' size, based on the properties of gold (position of the LA modes maximum, Fermi energy, sound velocity, etc.) are $\sim 100 \mathrm{~nm}$ length (of the nano-bar, or along the nano-ring) times the $\sim 3 \mathrm{~nm}$ thickness. An array of such identical objects, exposed to microwave radiation in as straightforward way (outlined in Ref. 11) as being insert into the cavity of a domestic microwave oven, is expected to produce a beam of $\mathrm{THz}$ radiation, which can be subsequently filtered and focused for practical use. We address the reader to Ref. 11 for the detailed analysis, as well as for citations of experimental works which provide some essential numerical estimates. We note that the use of gold as such is not a priori crucial for the success of the scheme, since LA 
phonons of the desired range exist in many materials. However, GNPs are routinely produced and used for different applications, and the electronic structure of gold can be fairly reasonably described within the framework of nearly-free-electron model, for the sake of numerical estimations done in Ref. 11.

\section{Terahertz-to-infrared converter}

This part deals with the problem of revealing the modified $\mathrm{THz}$ intensity, be it either in transmission or reflection geometry, to the observer. The approach suggested here is to create a $\mathrm{THz}$ "image" focused on an "active" surface, that will serve a source of secondary IR radiation. More precisely, an "active surface" would be a high-resolution matrix, every "element" of which would respond to incident $\mathrm{THz}$ radiation by generating heat. The secondary image created by the matrix will be then detected by a standard high-sensitive IR camera. The conversion into IR is chosen because it seems to be easiest to implement in practice. The suggested setup is specified in Fig. 1 for the transmission geometry and in Fig. 2 - for the reflection geometry. The parameters of commercially available IR cameras [12, 13] imply that the both schemes are realistic, in what concerns the resolution and sensitivity at the IR end. In the transmission mode (Fig. 1), the tissue sample areas containing cancer cells, rich in water, will strongly absorb the $\mathrm{THz}$ radiation, so that corresponding areas in the matrix will be in the $\mathrm{THz}$ "shadow" and thus come "cold" for the IR camera, on the background of image from healthy cells which let $\mathrm{THz}$ radiation pass and thus produce the "hot" image. In the reflection mode (Fig. 2), the IR coding will be inverted: the $\mathrm{THz}$ radiation source "illuminates" a body with the cancer tumour, whereas the tumour is also irradiated with the NIR laser. The laser's radiation penetrates the skin and tissue and excites 
surface plasmons in GNPs delivered in advance into the tumour. The surface plasmons heat the tumour, making it more reflective for the $\mathrm{THz}$ radiation. Therefore the $\mathrm{THz}$ image of the tumour will be more intense and thus "hot" for the IR camera.

We turn now to the design and properties of the THz-to-IR conversion unit. It is suggested to consist of sufficiently dense array of GNP deposited on, or distributed within, a buffer material, transparent to both $\mathrm{THz}$ and IR rays.

In a recent work [14], two of us studied a physical mechanism of heating the GNPs with radio frequency $(\mathrm{RF})$ radiation, whereby an essential role was played by longitudinal acoustic phonons. In the RF range and for the GNP sizes considered in Ref. [14], the radiation quantum was "too short" to excite an electron without an assistance from a phonon. The interplay of radiation, phonon bath and electron transitions between quantified levels of a GNP resulted in certain "rules" as concerning the efficiency of heating depending on the GNP sizes. The present case is in part similar, yet different in the sense that a $\mathrm{THz}$ quantum is much larger and can excite an electron without an intervention by an acoustic phonon, in what regards the energy conservation. The momentum conservation law is fulfilled due to uncertainty in momentum of the Fermi electrons of gold, as is discussed in the following Section.

\subsection{Absorption of THz photons by GNPs}

The key issues in "designing" the energy transfer between radiation, phonons and electrons are the conservation laws for energy and momentum, and other considerations affecting different relaxation scenarios for an excited electron. Ref. [11] deals with the process of (LA 
phonon + RF absorption $)-($ THz emission), depicted in Fig. 1 therein, and analyses the GNP shape favouring this scheme. In the present case, we deal with an almost opposite process, (THz absorption) - (LA phonon emission). The RF photon "helpful" in momentum conservation law does not come about here; however, we argue in the following that the momentum matching is achieved "by itself", within the accuracy imposed by the Heisenberg's uncertainty relation. If allowed by the conservation laws, emitting a phonon is "the most natural" relaxation channel for an excited electron and hence the "working cycle" of the THz-to-IR converter.

We proceed now with numerical estimations, assuming the frequency of the THz radiation source to be $4.2 \mathrm{THz}$, as from "our" $\mathrm{THz}$ source suggested in Ref. [11], tuned to the maximum of the LA vibration modes density in gold. The corresponding wavelength is 71.3 $\mu \mathrm{m}$, the energy and momentum of the THz photon, respectively, $h v=17.4 \mathrm{meV}$ and $p_{\mathrm{ph}}=h v /$ $c \approx 9.3 \cdot 10^{-25} \mathrm{~g} \cdot \mathrm{cm} \cdot \mathrm{s}^{-1}$ (from here on, $h$ is the Planck constant, and $c$ the speed of light in vacuum).

We need a nanoparticle with discrete electron levels that will be, at the Fermi level $E_{\mathrm{F}}$, in resonance with the THz photon energy $h v$. The estimation of the GNP spatial size (diameter $D$, assuming spherical shape) can be gained from the Kubo's formula for the level spacing at $E_{\mathrm{F}}$ (Refs. [15] and [16]; see also Appendix A in Ref. [14]):

$$
D=\left[(8 / \pi) \cdot\left(m_{\mathrm{a}} / \rho\right) \cdot\left(E_{\mathrm{F}} / h v\right)\right]^{1 / 3}
$$

This yields (specifying $m_{\mathrm{a}}$ for the atomic mass and $\rho$ for density) the GNPs diameter $D \approx 2.4$ $\mathrm{nm}$. The skin depth in gold at $4.2 \mathrm{THz}$ is about $36.4 \mathrm{~nm}$, hence exceeds the GNP size by more 
than an order of magnitude. For practical considerations, the electric field penetrates the volume and is of the same strength throughout the nanoparticle.

For the system of electron energy levels in resonance with $\mathrm{THz}$ photons thus established, one notes a mismatch of momenta on transition, since the slope of the dispersion relation for photons is very steep, and the corresponding cone $E_{\mathrm{ph}}\left(\mathbf{p}_{\mathrm{ph}}\right)$ is fully inside the $E(\mathbf{p})$ paraboloid for the electrons - see Fig. 3. The step in the electron momentum on transition upwards to the first vacant state above the Fermi level $E_{\mathrm{F}}$ is $\Delta p_{\mathrm{F}}=h v / \mathrm{vF}_{\mathrm{F}} \approx 2 \cdot 10^{-22} \mathrm{~g} \cdot \mathrm{cm} \cdot \mathrm{s}^{-1}$ (using the Fermi velocity in gold $\left.\mathrm{v}_{\mathrm{F}}=1.4 \cdot 10^{8} \mathrm{~cm} \cdot \mathrm{s}^{-1}[17]\right)$ and by two orders of magnitude larger than the photon's momentum which is $p_{\mathrm{ph}} \approx 9.3 \cdot 10^{-25} \mathrm{~g} \cdot \mathrm{cm} \cdot \mathrm{s}^{-1}$. However, in a particle of size $D$ the Heisenberg's uncertainty of momentum $\Delta p_{D}$ is correspondingly large, by force of the relation $\Delta p_{D} \cdot D \geq h / 2 \pi$, whence in our case $\Delta p_{D} \geq 4.4 \cdot 10^{-21} \mathrm{~g} \cdot \mathrm{cm} \cdot \mathrm{s}^{-1}$, i.e., at least $\approx 20$ times larger than the above momentum mismatch $\Delta p_{\mathrm{F}}$. This way, the situation is "saved" for a direct absorption of $\mathrm{THz}$ photons by the electron system (and the subsequent conversion of the energy into lattice vibrations, i.e., IR radiation).

\subsection{Modelling of heat exchange by GNP with its embedding substance}

The GNPs have to be fixed within, or at, the bearing matrix substance. Of possible materials for the latter, Teflon ${ }^{\circledR}$ seem convenient, because it yields high transmission in both IR and THz. In the following, some numerical estimates will be done for Teflon ${ }^{\circledR}$. According to the data of Ref. [18], the transmission of a Teflon film of $0.1 \mathrm{~mm}$ thickness at $71.3 \mu \mathrm{m}$ wavelength (i.e., at "our" $\mathrm{THz}$ photon's energy of $17.4 \mathrm{meV}$ ) is about $90 \%$, within the wavelength range 3 to $5 \mu \mathrm{m}$ - about $80-90 \%$, and within 9 to $12 \mu \mathrm{m}-$ about $50 \%$. These parameters indicate that the Teflon ${ }^{\circledR}$ film is applicable as a matrix material with sufficiently 
high transparency in both THz and IR wavelength ranges, whereby the usage of the Mirage 640 P-Series infrared camera [12] (specifically, a version with operating wavelength range from 3 to $5 \mu \mathrm{m}$ ) could be a good choice for the end detector. Another acceptable choice would be that of the FLIR A6700sc MWIR camera [13], equally with the operating wavelength range 3 to $5 \mu \mathrm{m}$.

The heat exchange of GNPs with the matrix in what regards the reaction time and "smearing" of the heat "element" over a larger spot in the matrix are important characteristics responsible for practical applications. A detailed analysis based on solving the heat transport equations will be given elsewhere [19]. Here, we show the solutions for a spherical GNP of radius $R_{0}=D / 2=1.2 \mathrm{~nm}$, embedded in quasi infinite medium (extending in the calculation up to the radius $R=500 \mathrm{~nm}$ ). These results would hopefully yield faithful estimates for the practical design we suggest, that is, an embedding of GNPs of $2.4 \mathrm{~nm}$ size into a $0.1 \mathrm{~mm}$ thick Teflon film.

We consider the time- and space-dependent evolution of temperature $T(r, t)$ throughout the composite system of gold sphere within the thick Teflon shell, solving the heat-transfer equation in spherical coordinates:

$$
\rho C \frac{\partial T}{\partial t}=\frac{1}{r^{2}} \frac{\partial}{\partial r}\left(\lambda r^{2} \frac{\partial T}{\partial r}\right)+q(r)
$$

where $\rho$ is density, $C$ : specific heat, $\lambda$ : specific heat conductivity, $q$ : volumic heat density function of the heat source, in our case assumed to be $q=$ [absorbed power]/[GNP volume]. The numerical solution is further on expressed as the deviation from the ambient temperature, $\Delta T=T-300 \mathrm{~K}$. The maximum (saturated) $\Delta T$ value, $\Delta T_{\mathrm{m}}$, is proportional to the absorbed power $Q$; the proportionality constant depends on the sizes and heat conductivities of gold 
and Teflon parts. Moreover, in order to account for practical imperfections, textures, etc. of GNPs, the "blackness", or emissivity factor $\alpha_{\mathrm{i}}$ is added, whose trial values were varied in broad limits from 1 to 0.1 . The ideal "blackness" $\alpha_{\mathrm{i}}=1$ assumes the black body radiation; lower values mean that a part of absorbed power will be lost for conversion into the IR, that is, the particle needs to be heated more to provide the same IR radiation yield. For practical estimations, if lacking more precise information about the emitting object, the emissivity factor $\alpha_{\mathrm{i}}=0.5$ is routinely assumed.

The results of simulation of the temperature evolution are shown in Fig. 4 as $\Delta T(t)$ at the GNPs centre (left panel) and as $\Delta T(r)$ in stationary regime (right panel). The five curves in each panel correspond, in ascending order, to $\alpha_{\mathrm{i}}=1$ through 0.1 and thus span the realistic range for this "sample-dependent" parameter. The time evolution (Fig.4, left panel) traces the increasing $\Delta T$ values up to their attaining the $99.5 \%$ of the saturation value $\Delta T_{\mathrm{m}}$, at which point the power was switched off, and the system cooled down.

The resulting on/off curves characterise the "reaction time" of the THz-to-IR converter, or otherwise the necessary duration of a $\mathrm{THz}$ irradiation necessary to fully develop the IR response. This time parameter (in fact quite dependent on the materials and sizes) was estimated as $\sim 75 \mathrm{~ns}$ for GNPs with diameter $D=2.4 \mathrm{~nm}$ in Teflon. It implies that, if the $\mathrm{THz}$ source is not operating permanently, the duration of its pulses should not be much shorter than the estimated "saturation time".

Fig. 4, right panel depicts the spatial distribution of the temperature increase inside and around the GNP; the particle radius is easily seen by a kink. The relevance of this information is the size of the "thermal spot" corresponding to a given temperature increase, and, consequently, the spatial resolution of the hot object as seen by the IR camera. Important is that the "smearing", in all cases (say, defined by the threshold value $\Delta T_{\mathrm{m}}=14 \mathrm{mK} / \alpha_{\mathrm{i}}=28 \mathrm{mK}$ 
at $\alpha_{\mathrm{i}}=0.5$ ) remains comparable to the GNP size and not spills over much larger spaces, deteriorating the "fine structure" of object in the IR vision.

\subsection{Sensitivity of IR detectors; GNP density within the matrix}

The manufacturers of IR cameras specify their temperature sensitivity $\Delta T_{\mathrm{bb}}$; however what is detected in fact is the energy flux $\Delta \varepsilon_{\mathrm{bb}}$ that falls onto the surface corresponding to a pixel of the IR camera detector matrix. For the Mirage 640 P-Series IR camera manufactured by Infrared Cameras Inc. [12], the reported thermal sensibility is $\Delta T_{\mathrm{bb}}=14 \mathrm{mK}$ (above the ambient background of $T=300 \mathrm{~K}$ ), and the pixel's size $d=15 \mu \mathrm{m}$. The calculations done for a GNP of the diameter $2.4 \mathrm{~nm}$ (skipped here; see details in Ref. 19) specify the absorbed power necessary to bring about the heating $\Delta T=\Delta T_{\mathrm{bb}} / \alpha_{\mathrm{i}}=28 \mathrm{mK}$ of a single GNP at $\alpha_{\mathrm{i}}=$ $0.5: Q_{\mathrm{i}}=0.11 \mathrm{nW}$.

We assume for the following the close up operation mode of the IR camera, so that the inspected surface element of the Teflon+GNPs layer is projected onto the IR camera detector in the 1:1 magnification. We would like to estimate the necessary minimal volume density of GNPs embedded in the Teflon layer to make them "visible". Out of GNPs embedded on a $d \times d$ surface element, only those situated within the depth of focus of the IR camera (typically, $l_{\text {dof }} \sim 0.3 \mathrm{~mm}$ ) will contribute; we consider in the following the Teflon layer thickness $\delta \leq$ $l_{\text {dof }}$, taking, for the sake of order-of-magnitude estimates, $\delta=0.1 \mathrm{~mm}$.

In order to use the full resolution power of IR detector, one should have at least one (better: more) GNP per surface element area. This sets the bottom limit on the GNP concentration: $N[\mathrm{GNP}] /$ Volume $=1 /\left(d^{2} \cdot \delta\right) \approx 4.4 \cdot 10^{4} \mathrm{~mm}^{-3}$. 


\section{Conclusion}

$\mathrm{THz}$ medical imaging promises to become a powerful non-invasive technique for monitoring the human skin's status for cancer, as well as for detecting pathological modifications in other organs. In this connection, the development of THz imaging setups is one of the "hottest" areas in nanotechnology-supported modalities for the cancer diagnostics. We put forward an idea of how a THz emitter in combination with a THz-to-IR converter, the latter being equipped with highly-sensitive IR camera, could lay foundation for a practical $\mathrm{THz}$ cancer imaging. Gold nano-objects play the crucial role in both elements of this setup, although their shape/size and the functionality are different. The $\mathrm{THz}$ emitter - in fact, a (previously described and here only briefly mentioned) converter of $\mathrm{THz}$ lattice vibrations into $\mathrm{THz}$ radiation - employs gold nano-bars or nano-rings; the energy can be provided by a domestic microwave oven. The (newly introduced here) THz-to-IR converter needs an efficient, strongly localised and rapidly responding energy transfer mechanism from $\mathrm{THz}$ radiation into heat; the essential role here is supposed to be taken over by gold nano-spheres (of $2.4 \mathrm{~nm}$ diameter) embedded into a thin Teflon film. Numerical calculations suggest the performance of the conversion to be sufficient for enabling an use of available commercial IR cameras as terminal detectors. Estimated heating/cooling times of $2.4 \mathrm{~nm}$ diameter gold nano-spheres in the Teflon matrix ( $75 \mathrm{~ns})$ show that $(i)$ the considered THz-to-IR converter could be operational on a real-time scale; (ii) to run it, one needs either a continuous or longer-than-75 ns pulsed $\mathrm{THz}$ source. The above mentioned "domestic" $\mathrm{THz}$ radiation source would be a possible choice. Finally, we estimated the minimal concentration of GNPs in the Teflon film $\left(4.4 \cdot 10^{4} \mathrm{~mm}^{-3}\right)$ for combining good sensitivity and spatial resolution. 
This design opens the way to attempts of practical implementation, whereby an elaboration of the process for making the Teflon ${ }^{\circledR}$ film containing gold nanoparticles has yet to be solved.

\section{References}

1. Hu B.B., Nuss M.C. Imaging with terahertz waves. Opt. Lett. 1995, 20(16), 1716.

2. Ross K.F., Gordon R.E. Water in malignant tissue, measured by cell refractometry and nuclear magnetic resonance. J. Microsc. 1982, 128, 7-21.

3. Chen J.H., Avram H.E., Crooks L.E., et al. In vivo relaxation times and hydrogen density at 0.063-4.85 T in rats with implanted mammary adenocarcinomas. Radiology 1992, 184, 427-434.

4. Berry E., Walker G.C., Fitzgerald A.J., et al. Do in vivo terahertz imaging systems comply with safety guidelines? J. Laser Appl. 2003, 15(3), 192-198.

5. Rønne C., Thrane L., Åstrand P.O., et al. Investigation of the temperature dependence of dielectric relaxation in liquid water by $\mathrm{THz}$ reflection spectroscopy and molecular dynamics simulation. J. Chem. Phys. 1997, 107, 5319-5331. 
6. Oh S.J., Maeng I., Hee J.S., et al. Nanoparticle contrast agents for terahertz medical imaging. 33rd international conference on infrared and millimeter waves and the 16 th international conference on terahertz electronics, 2008, IRMMW-THz 2008; 15-19 September 2008; Pasadena, CA; 2008.

7. Oh S.J., Kang J., Maeng I., et al. Nanoparticle-enabled terahertz imaging for cancer diagnosis. Opt Express. 2009, 17(5), 3469-3475.

8. Oh S.J., Choi J., Maeng I., et al. High-sensitivity terahertz imaging technique using nanoparticle probes for medical applications. 2010 IEEE photonics society winter topicals meeting, WTM 2010; 11-13 January 2010; Majorca; 2010; 52-53.

9. Oh S.J., Choi J., Maeng I., et al. Molecular imaging with terahertz waves. Opt Express. 2011, 19(5), 4009-4016.

10. Oh S.J., Huh Y.-M., Suh J.-S., et al. Cancer Diagnosis by Terahertz Molecular Imaging Technique. Journal of Infrared, Millimeter and Terahertz Waves 2012, 33, 74-81.

11. Moldosanov K. and Postnikov A. A terahertz-vibration to terahertz-radiation converter based on gold nanoobjects: a feasibility study, Beilstein J. Nanotechnol. 2016, 7, 983-989.

12. Infrared Cameras Inc., "Mirage P-Series Infrared Thermal Imaging Camera," http:// www.infraredcamerasinc.com/pages/mirage640-p-series-fixed-mount-scientific-electricalsecurity-infrared-camera.html . 
13. FLIR Systems, Inc., http://www.flir.com/instruments/display/?id=56784, http:// www.flir.com/instruments/display/?id=62960.

14. Postnikov A., Moldosanov K. Phonon-Assisted Radiofrequency Absorption by Gold Nanoparticles Resulting in Hyperthermia. In Fundamental and Applied NanoElectromagnetics; Maffucci A., Maksimenko S. A., Eds.; NATO Science for Peace and Security Series B: Physics and Biophysics; Springer: Dordrecht, The Netherlands, 2016; pp 171-201. doi: 10.1007/978-94-017-7478-9_9 . Preprint: http://arxiv.org/abs/ 1508.00735 .

15. Kubo R. Electronic properties of metallic fine partiles. I., J. Phys. Soc. Jpn. 1962, 17, 975-986. doi: 10.1143/JPSJ.17.975

16. Kubo R. Discreteness of energy levels in small metallic particles, J. Phys. Colloques 1977, 38, C2-69-C2-75. doi: 10.1051/jphyscol:1977214

17. Ashcroft N.W.; Mermin N.D. Solid State Physics; Saunders College: Henrietta, New York, U.S.A., 1976.

18. TYDEX ${ }^{\circledR}$, J.S.Co., "THz Materials," in http://www.tydexoptics.com/en/products/ thz_optics/thz_materials/. 
19. Moldosanov K.A., Kairyev N.J., Lelevkin V.M. and Postnikov A.V. Prospects for terahertz imaging the human skin cancer with the help of the gold-nanoparticle-based terahertz-to-infrared converter, to be published. 


\section{Figure captions}

Figure 1: Transmission mode for in vitro studies: 1 - a tissue sample, 2 - objective (high resistivity float zone silicon, high density polyethylene or Teflon ${ }^{\circledR}$ ), 3 - THz-to-IR-converter, 4 - highly sensitive IR camera.

Figure 2: Reflection mode for in vivo imaging with a usage of the near-infrared (NIR) laser for excitation of surface plasmons in GNPs inside a tumour (for heating water in cancer cells): 1 - tumour, 2, 3, 4- the same as in Figure 1.

Figure 3: Scheme of absorption of THz photon by the Fermi electron. Dashed curve indicates a transition of the Fermi electron excited by the THz photon with energy $h v$.

Figure 4: Calculated time response to heating (left panel) and radial temperature profile (right panel) of the GNPs of radius $R_{0}=1.2 \mathrm{~nm}$ embedded in Teflon ${ }^{\circledR}$ spherical shell of effectively infinite radius $R=500 \mathrm{~nm}$. The five curves from the lower one (saturating to / departing from $\Delta T=14 \mathrm{mK}$ ) through the upper one (saturating to / departing from $\Delta T=140 \mathrm{mK}$ ) correspond to the values of the emissivity factor $\alpha_{\mathrm{i}}=1.0,0.7,0.5,0.3,0.1$. See text for explanations. 


\section{Figures}

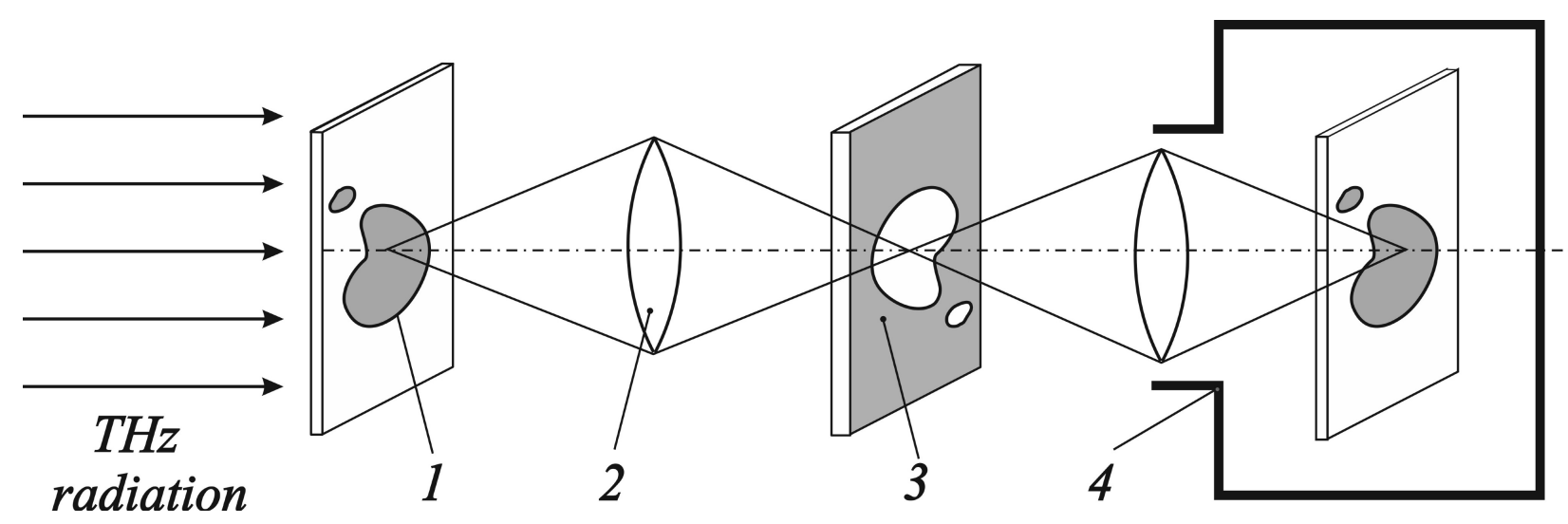

Figure 1: Transmission mode for in vitro studies: 1 - a tissue sample, 2 - objective (high resistivity float zone silicon, high density polyethylene or Teflon ${ }^{\circledR}$ ), 3 - THz-to-IR-converter, 4 - highly sensitive IR camera.

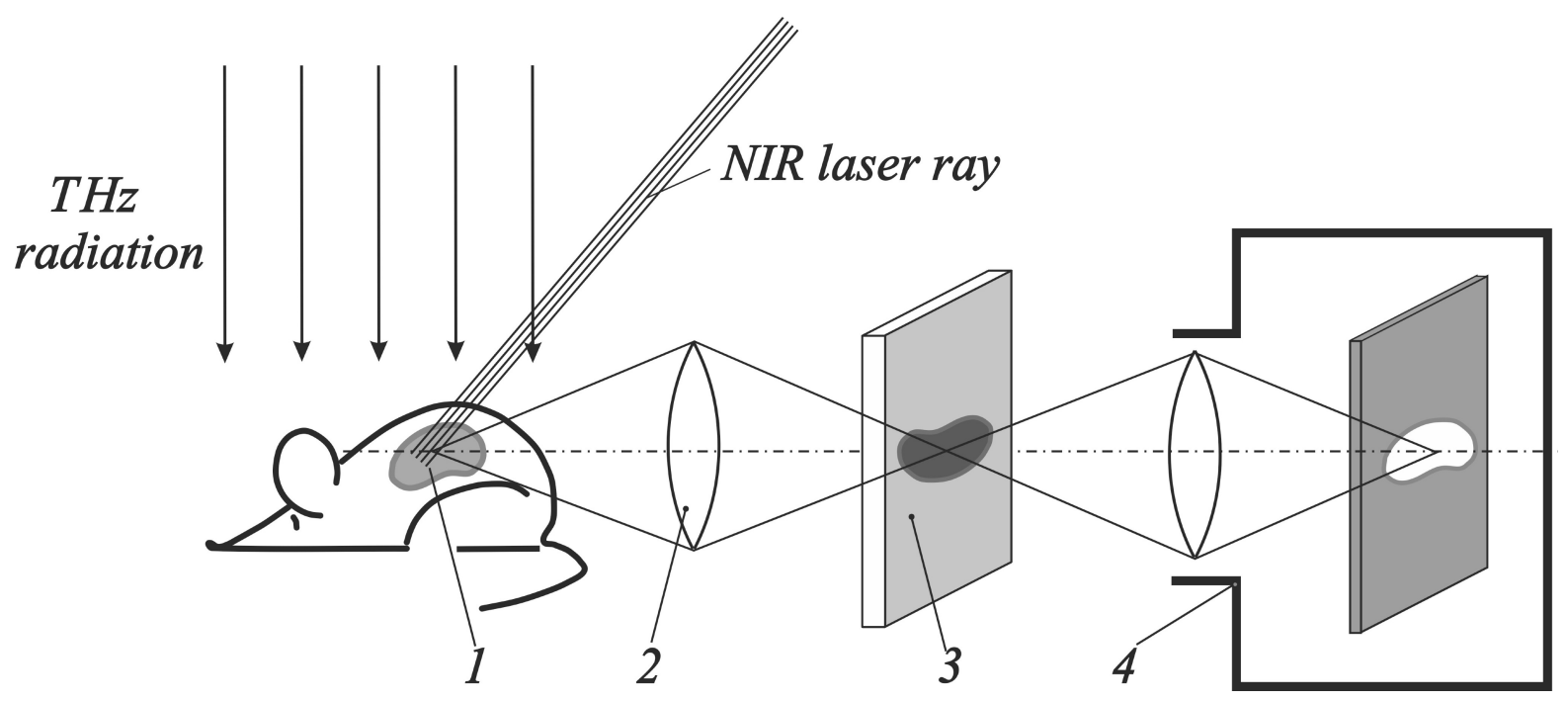

Figure 2: Reflection mode for in vivo imaging with a usage of the near-infrared (NIR) laser for excitation of surface plasmons in GNPs inside a tumour (for heating water in cancer cells): 1 tumour, 2, 3, 4- the same as in Figure 1. 


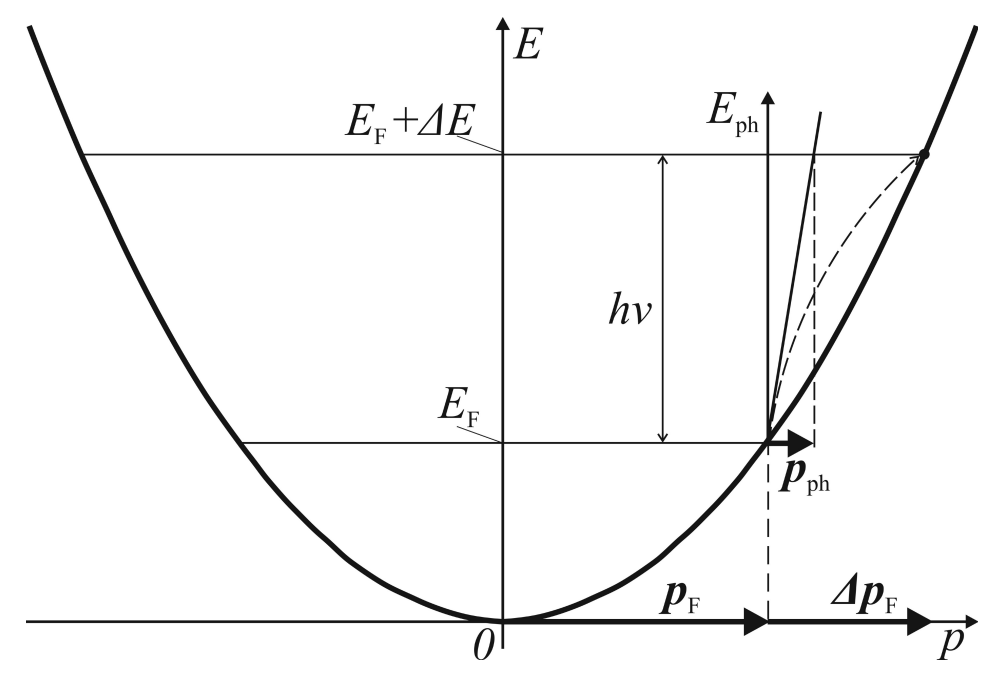

Figure 3: Scheme of absorption of THz photon by the Fermi electron. The dashed curve indicates a transition of the Fermi electron excited by the THz photon with energy $h v$.
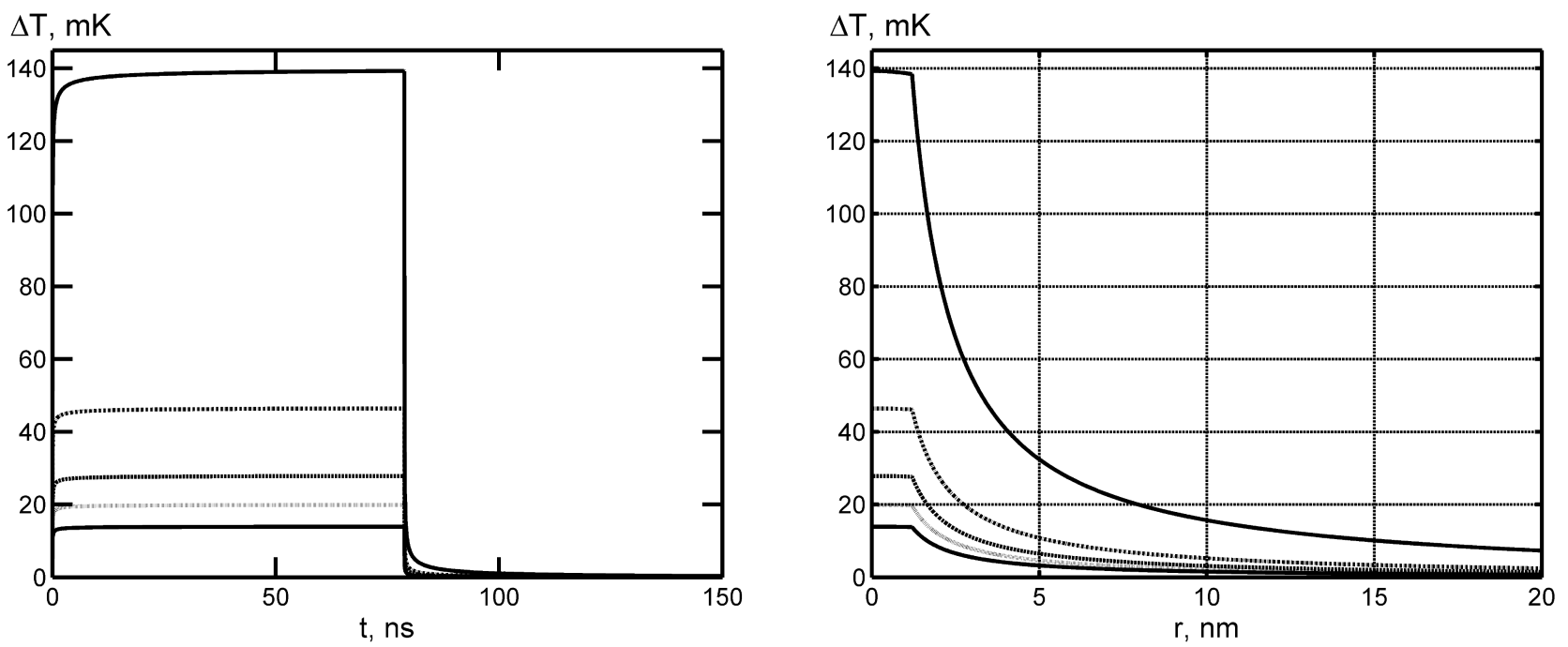

Figure 4: Calculated time response to heating (left panel) and radial temperature profile (right panel) of the GNPs of radius $R_{0}=1.2 \mathrm{~nm}$ embedded in Teflon ${ }^{\circledR}$ spherical shell of effectively infinite radius $R$ $=500 \mathrm{~nm}$. The five curves from the lower one (saturating to / departing from $\Delta T=14 \mathrm{mK}$ ) through the upper one (saturating to / departing from $\Delta T=140 \mathrm{mK}$ ) correspond to the values of the emissivity factor $\alpha_{i}=1.0,0.7,0.5,0.3,0.1$. See text for explanations. 